\title{
Newcastle University
}

\section{Newcastle University ePrints}

\author{
Thomas KS, Crook AM, Nunn AJ, Foster KA, Mason JM, Chalmers JR, Nasr IS, \\ Brindle RJ, English J, Meredith SK, Reynolds NJ, de Berker D, Mortimer PS, \\ Williams HC. Penicillin to Prevent Recurrent Leg Cellulitis. New England \\ Journal of Medicine 2013, 368(18), 1695-1703.
}

\section{Copyright:}

From New England Journal of Medicine, Thomas KS, Crook AM, Nunn AJ, Foster KA, Mason JM, Chalmers JR, Nasr IS, Brindle RJ, English J, Meredith SK, Reynolds NJ, de Berker D, Mortimer PS, Williams HC. Penicillin to Prevent Recurrent Leg Cellulitis. Volume 368 No. 18.

Copyright (C) 2013 Massachusetts Medical Society. Reprinted with Permission.

DOI link to article:

http://dx.doi.org/10.1056/NEJMoa1206300

Date deposited: 22-07-2014

ePrints - Newcastle University ePrints

http://eprint.ncl.ac.uk 
ORIGINAL ARTICLE

\section{Penicillin to Prevent Recurrent Leg Cellulitis}

\author{
Kim S. Thomas, Ph.D., Angela M. Crook, Ph.D., Andrew J. Nunn, M.Sc., \\ Katharine A. Foster, Ph.D., James M. Mason, D.Phil., Joanne R. Chalmers, Ph.D., \\ Ibrahim S. Nasr, M.Sc., Richard J. Brindle, D.M., John English, M.B., B.S., \\ Sarah K. Meredith, F.F.P.H., Nicholas J. Reynolds, M.D., F.R.C.P., \\ David de Berker, M.D., F.R.C.P., Peter S. Mortimer, M.D., F.R.C.P., \\ and Hywel C. Williams, Ph.D., F.R.C.P., for the U.K. Dermatology \\ Clinical Trials Network's PATCH I Trial Team*
}

A BSTRACT
BACKGROUND

Cellulitis of the leg is a common bacterial infection of the skin and underlying tissue. We compared prophylactic low-dose penicillin with placebo for the prevention of recurrent cellulitis.

\section{METHODS}

We conducted a double-blind, randomized, controlled trial involving patients with two or more episodes of cellulitis of the leg who were recruited in 28 hospitals in the United Kingdom and Ireland. Randomization was performed according to a computer-generated code, and study medications (penicillin [250 mg twice a day] or placebo for 12 months) were dispensed by a central pharmacy. The primary outcome was the time to a first recurrence. Participants were followed for up to 3 years. Because the risk of recurrence was not constant over the 3-year period, the primary hypothesis was tested during prophylaxis only.

\section{RESULTS}

A total of 274 patients were recruited. Baseline characteristics were similar in the two groups. The median time to a first recurrence of cellulitis was 626 days in the penicillin group and 532 days in the placebo group. During the prophylaxis phase, 30 of 136 participants in the penicillin group (22\%) had a recurrence, as compared with 51 of 138 participants in the placebo group (37\%) (hazard ratio, 0.55; 95\% confidence interval $[\mathrm{CI}], 0.35$ to $0.86 ; \mathrm{P}=0.01$ ), yielding a number needed to treat to prevent one recurrent cellulitis episode of 5 (95\% CI, 4 to 9). During the no-intervention follow-up period, there was no difference between groups in the rate of a first recurrence ( $27 \%$ in both groups). Overall, participants in the penicillin group had fewer repeat episodes than those in the placebo group (119 vs. $164, \mathrm{P}=0.02$ for trend). There was no significant between-group difference in the number of participants with adverse events ( 37 in the penicillin group and 48 in the placebo group, $\mathrm{P}=0.50$ ).
From the Centre of Evidence-Based Dermatology, University of Nottingham (K.S.T., K.A.F., J.R.C., H.C.W.), and the Department of Dermatology, Nottingham University Hospitals National Health Service Trust (J.E.), Nottingham; the Medical Research Council Clinical Trials Unit (A.M.C., A.J.N., S.K.M.) and the Division of Clinical Sciences, St. George's University of London (P.S.M.), London; the School of Medicine, Pharmacy, and Health, Durham University, Durham (J.M.M.); the Department of Dermatology, Royal Berkshire Hospital, Reading (I.S.N.); the Departments of Microbiology (R.J.B.) and Dermatology (D.B.), Bristol Royal Infirmary, Bristol; and Newcastle University, Newcastle upon Tyne (N.J.R.) - all in the United Kingdom. Address reprint requests to Dr. Williams at the Centre of EvidenceBased Dermatology, University of Nottingham, King's Meadow Campus, Lenton Ln., Nottingham NG7 2NR, United Kingdom, or at hywel.williams@nottingham.ac.uk.

* Members of the Prophylactic Antibiotics for the Treatment of Cellulitis at Home I (PATCH I) Trial Team are listed in the Supplementary Appendix, available at NEJM.org.

N EnglJ Med 2013;368:1695-703. DOI: 10.1056/NEJMoal206300

Copyright @ 2013 Massachusetts Medical Society.

\section{CONCLUSIONS}

In patients with recurrent cellulitis of the leg, penicillin was effective in preventing subsequent attacks during prophylaxis, but the protective effect diminished progressively once drug therapy was stopped. (Funded by Action Medical Research; PATCH I Controlled-Trials.com number, ISRCTN34716921.) 
ELLULITIS OF THE LEG IS A COMMON INfection of the skin and subcutaneous tissue. $^{1,2}$ Most infections that affect intact skin are thought to be due to streptococci, ${ }^{3,4}$ although other organisms may be responsible if the integrity of the skin is compromised. Each recurrent episode of cellulitis results in further damage to the lymphatic system ${ }^{5,6}$ and is associated with additional morbidity and health care costs. $^{7}$ Current guidelines for the prevention of cellulitis are consensus-based and recommend prophylactic antibiotic therapy for recurrent disease. ${ }^{8,9}$ Three small, randomized, controlled trials have suggested a possible benefit of prophylactic antibiotics in patients with recurrent cellulitis, ${ }^{10-12}$ but clinical opinion is mixed.

We conducted the Prophylactic Antibiotics for the Treatment of Cellulitis at Home I (PATCH I) trial to examine the effectiveness of a 12-month course of low-dose penicillin for the prevention of cellulitis of the leg in persons with recurrent disease. This study builds on data from a previous study of 6 months of penicillin prophylaxis, primarily in patients with a first episode of cellulitis of the leg. ${ }^{2}$

\section{METHODS}

\section{TRIAL DESIGN AND OVERSIGHT}

This double-blind, randomized, controlled trial compared 12 months of prophylactic phenoxymethylpenicillin (penicillin) with placebo in patients with recurrent cellulitis. Participants were monitored for up to 36 months (minimum, 18). Changes to the trial protocol after the start of recruitment included the following: the number of repeat episodes of cellulitis was added as a secondary outcome, since this outcome had been erroneously omitted; and to boost recruitment, the window between the end of the index episode and recruitment was extended from 3 to 6 months and potential participants were sought through direct advertising to the public. For full details of the trial design, see the protocol, available with the full text of this article at NEJM.org.

Appropriate national ethics and regulatory approvals were obtained; all participants gave written informed consent. The Medical Research Council Clinical Trials Unit provided input into the design and conduct of the trial and performed the statistical analysis. All the authors vouch for the completeness and accuracy of the data presented and for the fidelity of the study to the protocol. There was no commercial support for this study.

\section{PARTICIPANTS}

Recruitment took place at 28 hospitals in the United Kingdom and Ireland between July 2006 and January 2010 (date of last follow-up contact, July 2011). Participants were identified in a hospital setting or through direct advertising.

Patients who had had a recurrent episode of leg cellulitis within the previous 24 weeks were eligible for inclusion. Patients were considered to have recurrent cellulitis if they had had at least two episodes of cellulitis of the leg within the previous 3 years (as assessed by the recruiting dermatologist on the basis of a history taking and clinical examination). If a patient was not assessed by a dermatologist during the acute episode, the diagnosis was confirmed on the basis of the patient's medical records at the recruiting hospital in combination with an interview with the patient. The following were required: local warmth, tenderness, or acute pain; unilateral erythema or bilateral erythema, with a temporal association between symptoms and the more severely affected leg; and unilateral edema. If there was doubt about the certainty of the diagnosis, the patient was excluded.

Other exclusion criteria were the use of antibiotics for the prevention of cellulitis in the preceding 6 months; allergy to penicillin; previous leg ulceration, surgery, or penetrating trauma; an unwillingness of the recruiting clinician to randomly assign the patient for medical reasons; an age of less than 16 years; an inability to give informed consent; and current participation in another clinical trial.

\section{INTERVENTIONS}

Participants received low-dose oral penicillin (250 mg) or placebo (consisting of calcium phosphate, starch, cellulose, and magnesium stearate) twice daily after completion of treatment for the index episode of cellulitis. Normal clinical practice was observed for the treatment of predisposing factors such as tinea pedis.

Adherence to trial medication was assessed by means of self-reported pill counts collected during follow-up telephone calls. These data were categorized as no pills taken, hardly any taken ( 1 to $24 \%$ of prescribed doses), some taken (25 to $49 \%$ ), most taken (50 to $74 \%$ ), or all taken (75 to $100 \%$ ). 


\section{RANDOMIZATION, BLINDING, AND FOLLOW-UP}

The coordinating center randomly assigned the participants with the use of the Nottingham Clinical Trials Unit (NCTU) Web-based randomization service. Randomization was stratified according to the presence or absence of preexisting edema and of ulceration associated with the cellulitis. The computer-generated randomization list was produced before the start of recruitment, with the use of randomly varying block sizes, and was held by the NCTU. Treatment assignments were sent electronically to the pharmacy department at Queen's Medical Centre, Nottingham, where the medications were dispensed with the use of identical labeling and packaging and were mailed to the participants' homes.

Participants and all members of the study team were unaware of the treatment assignments throughout the trial, and the analysis was performed before the breaking of the randomization code. Although the study medications were packaged in an identical way and the placebo tablets were the same size and shape as the penicillin tablets, the tablets were not identical owing to the cost implications of overencapsulation (the placebo tablets were unmarked, and the penicillin tablets were marked). The risk of unblinding by direct comparison of active and placebo tablets was low, because participants were recruited from a wide geographic area, with little or no contact with each other. In addition, the primary outcome was confirmed on the basis of medical records kept by general practitioners in order to reduce potential detection bias from unblinding.

Follow-up telephone calls from the coordinating center were conducted at 3-month intervals during the prophylaxis phase ( 0 to 12 months) and at 6-month intervals during the follow-up phase (13 to 36 months). Participants recorded adverse events and the use of health services in a diary.

\section{OUTCOMES}

The primary outcome measure was the time from randomization to the next medically confirmed episode of cellulitis. The episode was considered to have started on the first day of symptoms reported by the participant. Episodes reported by the participant and resulting in antibiotic treatment but not confirmed by a medical professional were documented as self-confirmed cases and included in the sensitivity analysis.
Secondary outcome measures were the proportions of participants with a repeat episode of cellulitis during the prophylaxis phase and during the follow-up phase, the number of repeat episodes of cellulitis, the proportions of participants with new edema or ulceration during the prophylaxis phase and during the follow-up phase, the number of nights in the hospital for cellulitis, the number of adverse drug reactions or adverse events of interest (death, nausea, diarrhea, thrush, rash, severe skin reactions, sepsis, and renal failure), and cost-effectiveness. In addition, predictors of response were included as secondary outcomes in order to explore the effect of known risk factors on prediction of the efficacy of prophylaxis.

\section{STATISTICAL ANALYSIS}

We assumed a $50 \%$ reduction in the recurrence rate with penicillin as compared with placebo on the basis of a log-rank test for time-to-event data, with $80 \%$ power at a two-sided significance level of $5 \%$ and an expected $20 \%$ rate of loss to follow-up. Previous studies have shown possible recurrence rates of 30 to $50 \%$, depending on the population and the duration of follow-up. ${ }^{13,14}$ For the relapse rate in the placebo group, we used a conservative estimate of $35 \%$ over a period of 3 years. These calculations resulted in a sample size of 260 participants.

All analyses were prespecified in the statistical analysis plan. Analysis of the primary outcome included all randomly assigned participants, with no exclusions (intention-to-treat population). The time to recurrence was analyzed with the use of a Cox proportional-hazards model, and participants with limited follow-up data were included in the analysis but with data censored accordingly. The primary analysis was unadjusted, but the results of an analysis adjusted for stratification factors are also presented. Because the assumption of a constant hazard over time was not met $(\mathrm{P}=0.05)$, it was necessary to limit the piecewise Cox proportional-hazards model to the 12-month prophylaxis period for the primary analysis. ${ }^{15}$ The end of the prophylaxis period was prespecified in the statistical analysis plan as being a logical cutoff point for such a piecewise model. Results of an analysis of the 2-year and 3-year follow-up data are presented but should be considered supportive of the primary analysis. In addition, five sensitivity analyses of the primary outcome were conducted: episodes of self-confirmed cellulitis recurrences 
were included; participants who did not start the study medication or who reported a relapse within 4 weeks after randomization were excluded because incomplete treatment of the index episode was likely; participants who had received treatment for the index episode more than 12 weeks before randomization were excluded (a protocol change); patients with recurrences that were preceded by ulceration or trauma (in line with inclusion criteria) were excluded; and patients were stratified according to recruitment source (hospital or community).

For the secondary outcomes, differences in proportions of participants were compared with the use of the chi-square test, and differences in the proportion of participants reporting multiple episodes were compared with the use of the MannWhitney rank-sum test. A logistic-regression model was developed to explore factors associated with the failure of prophylaxis (defined as at least one confirmed episode of cellulitis during the prophylaxis phase). All baseline factors listed in Table 1 were included in the model, plus the interval between the index episode of cellulitis and randomization.

Undiscounted costs of care were estimated by applying published national reference $\operatorname{costs}^{16-18}$ to the use of resources. Confidence intervals were estimated with the use of bootstrapping methods, with 10,000 replications for each item. ${ }^{19}$ Two cost estimates are provided: the cost of U.K. National Health Service (NHS) resources alone and the overall societal cost, which included the cost of time lost from work or daily activities. All statistical analyses were conducted with the use of Stata software, version 11.2 (StataCorp).

RESULTS

\section{PARTICIPANTS}

Recruitment took place from July 2006 through January 2010. Of 533 patients screened, 274 were eligible and gave written informed consent; 136 were assigned to the penicillin group, and 138 to the placebo group (Fig. 1). Of these patients, 15 (5\%) withdrew consent or were lost to follow-up and 11 (4\%) died. A total of 206 patients (75\%) were recruited in secondary care. The baseline characteristics of the participants were well balanced between the groups (Table 1).

A total of 247 patients $(90 \%)$ underwent at least 18 months of follow-up (median, 25). Slower-than- anticipated recruitment meant that follow-up was limited for those recruited toward the end of the trial. A total of 214 participants (78\%) reported taking at least $75 \%$ of the study tablets; the proportion of patients who reported taking at least $75 \%$ of the tablets was similar in the two groups (79\% in the penicillin group and $78 \%$ in the placebo group).

\section{PRIMARY OUTCOME}

The median time to the first confirmed recurrence of cellulitis was 626 days in the penicillin group and 532 days in the placebo group. During the prophylaxis phase, 30 of 136 participants who received penicillin (22\%) had a recurrence, as compared with 51 of 138 participants who received placebo (37\%). Results from the Cox proportionalhazards model are summarized in Table 2.

During the prophylaxis phase, participants in the penicillin group had a $45 \%$ reduction in the risk of a repeat episode of cellulitis, as compared with those in the placebo group (hazard ratio, 0.55 ; $95 \%$ confidence interval [CI], 0.35 to 0.86 ; $\mathrm{P}=0.01$ ). This is equivalent to an absolute difference in event rates of 15 percentage points and a number needed to treat to prevent one repeat episode of 5 (95\% CI, 4 to 9). However, this significant effect was not sustained after prophylaxis ceased (Table 2 and Fig. 2). The results of all sensitivity analyses were consistent with the primary result.

\section{SECONDARY OUTCOMES}

The proportions of patients with a repeat episode of cellulitis during the prophylaxis phase and during the follow-up phase are shown in Table 2. Of the 129 participants with at least one confirmed repeat episode, 50 (39\%) had one repeat episode, 38 (29\%) had two, 20 (16\%) had three, and 21 (16\%) had four or more. Overall, participants in the penicillin group had fewer repeat episodes than those in the placebo group (119 vs. $164, \mathrm{P}=0.02$ for trend). During the prophylaxis phase, there were 76 repeat episodes in the penicillin group, as compared with 122 in the placebo group $(\mathrm{P}=0.03)$. During the follow-up phase, there were 43 and 42 repeat episodes in the penicillin and placebo groups, respectively $(\mathrm{P}=0.88)$.

A total of 89 participants (32\%) had no edema or ulceration at baseline, but 54 had these symptoms during the trial. There were no significant between-group differences in the development of 


\begin{tabular}{|c|c|c|}
\hline Characteristic & $\begin{array}{l}\text { Penicillin } \\
(\mathrm{N}=136)\end{array}$ & $\begin{array}{l}\text { Placebo } \\
(\mathrm{N}=138)\end{array}$ \\
\hline \multicolumn{3}{|l|}{$\begin{array}{l}\text { Preexisting leg edema or ulceration associated with cellulitis - } \\
\text { no. of patients (\%) }\end{array}$} \\
\hline Neither & $45(33)$ & $44(32)$ \\
\hline Edema & $81(60)$ & $82(59)$ \\
\hline Ulceration & $1(1)$ & $2(1)$ \\
\hline Both & $9(7)$ & $10(7)$ \\
\hline \multicolumn{3}{|l|}{ Age $-y r$} \\
\hline Mean & $58.1 \pm 12.6$ & $57.4 \pm 14.4$ \\
\hline Median (interquartile range) & $59(50-65)$ & $58(46-69)$ \\
\hline Female sex - no. of patients (\%) & $83(61)$ & $82(59)$ \\
\hline White race and British nationality — no. of patients (\%) & $115(85)$ & $121(88)$ \\
\hline \multicolumn{3}{|l|}{ No. of previous cellulitis episodes } \\
\hline Mean & $3.7 \pm 4.3$ & $3.8 \pm 4.8$ \\
\hline Median (interquartile range) & $2(1-5)$ & $2(1-4)$ \\
\hline Local warmth, tenderness, or acute pain — no. of patients (\%) & $136(100)$ & $138(100)$ \\
\hline Erythema at the affected site - no. of patients (\%) & $135(99)$ & $136(99)$ \\
\hline Edema at the affected site - no. of patients (\%) & $135(99)$ & $138(100)$ \\
\hline \multicolumn{3}{|l|}{ BMI } \\
\hline Mean & $35.1 \pm 9.4$ & $35.2 \pm 9.5$ \\
\hline Median (interquartile range) & $33.7(27.7-38.9)$ & $32.5(27.8-40.7)$ \\
\hline \multicolumn{3}{|l|}{ Chronic edema — no. of patients $(\%) \dagger$} \\
\hline Asymmetric & $64(47)$ & $64(46)$ \\
\hline Symmetric & $28(21)$ & $28(20)$ \\
\hline Venous insufficiency — no. of patients (\%) & $36(26)$ & $34(25)$ \\
\hline Leg ulceration subsequent to cellulitis — no. of patients (\%) $\dagger$ & $13(10)$ & $12(9)$ \\
\hline Tinea pedis or toe-web maceration — no. of patients (\%) & $52(38)$ & $48(35)$ \\
\hline Surgery $>2$ wk before the index cellulitis episode - no. of patients (\%) & $22(16)$ & $18(13)$ \\
\hline Blunt injury — no. of patients (\%) & $6(4)$ & $11(8)$ \\
\hline Definite or possible onychomycosis — no. of patients (\%) & $30(22)$ & $39(28)$ \\
\hline $\begin{array}{l}\text { Inpatient admission for index episode of cellulitis at baseline - } \\
\text { no. of patients (\%) }\end{array}$ & $65(48)$ & $59(43)$ \\
\hline Duration of hospital stay for hospitalized participants - days & $7.7 \pm 5.7$ & $5.7 \pm 4.3$ \\
\hline
\end{tabular}

* Plus-minus values are means \pm SD. No significant between-group differences were observed at baseline. BMI denotes body-mass index, calculated as the weight in kilograms divided by the square of the height in meters.

$\uparrow$ The values for chronic edema and leg ulceration at baseline vary slightly from the values for the stratification variables (preexisting leg edema or ulceration associated with cellulitis) as a result of the different data-collection methods used.

edema or ulceration during either the prophylaxis phase $(40 \%$ in the penicillin group and $48 \%$ in the placebo group, $\mathrm{P}=0.46$ ) or the follow-up phase $(40 \%$ and $45 \%$, respectively; $\mathrm{P}=0.60)$.

\section{SAFETY}

During the prophylaxis phase, one or more adverse events of prespecified interest occurred in
85 participants (37 in the penicillin group and 48 in the placebo group, $\mathrm{P}=0.50$ ) (see Table $\mathrm{S} 1$ in the Supplementary Appendix, available at NEJM.org). Eleven participants died during the trial $(8$ in the penicillin group and 3 in the placebo group, $\mathrm{P}=0.14)$; none of the deaths were considered to be related to the study drugs (Table $\mathrm{S} 1$ in the Supplementary Appendix). 


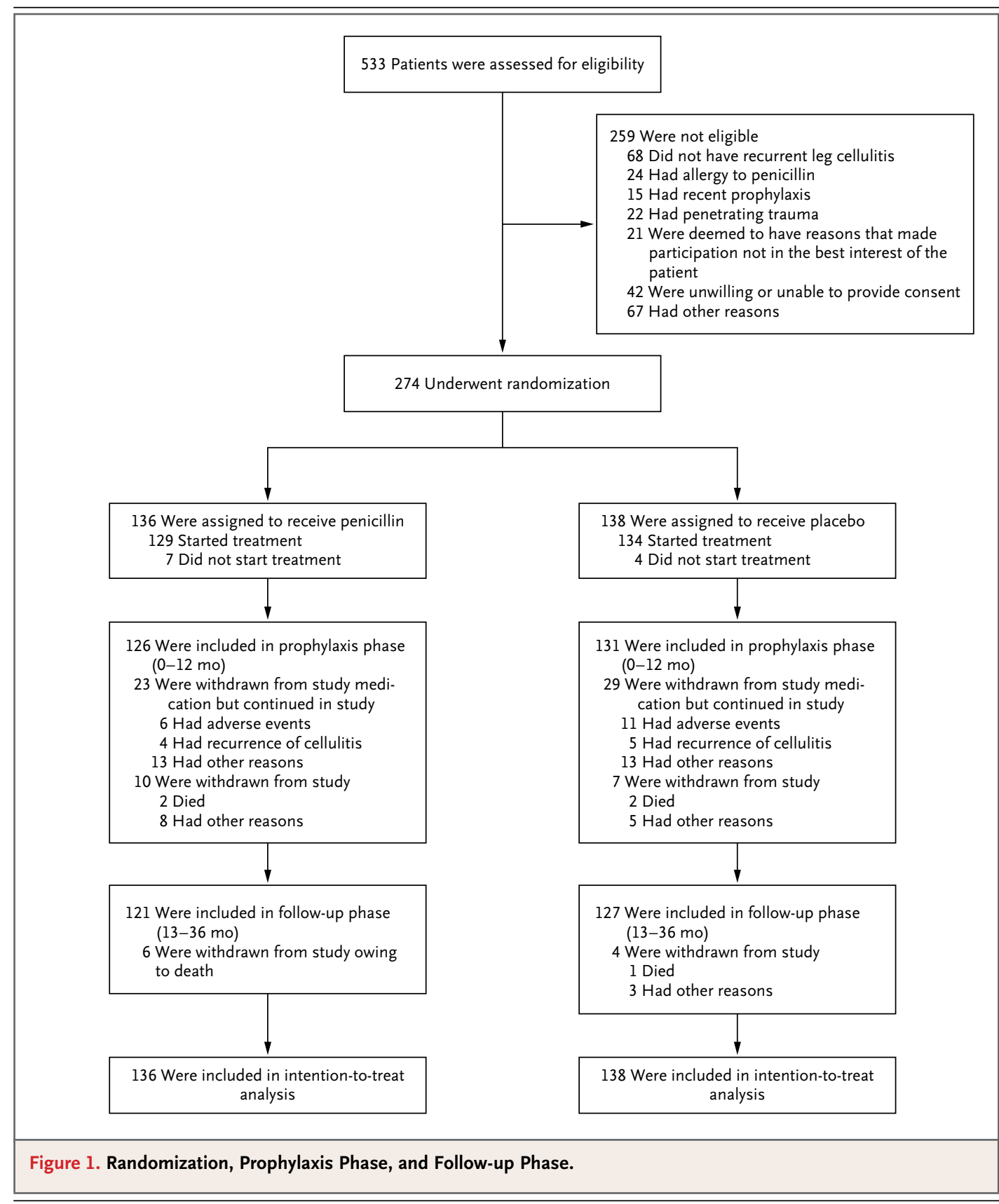

\section{PREDICTORS OF PROPHYLAXIS FAILURE}

Univariable and multivariable analyses of data from the 12-month prophylaxis period were performed to identify factors that might predict prophylaxis failure. The following factors were significantly associated with a poor response to treatment: a body-mass index (BMI; the weight in kilograms divided by the square of the height in meters) of 33 or higher, three or more previous episodes of cellulitis, and the presence of edema (borderline significance) (Table 3).

\section{RESOURCE USE AND COST}

There were no significant between-group differences in the use of health care services or in costs (Table S2 in the Supplementary Appendix). The difference in (undiscounted) NHS costs (perpatient cost in the penicillin group minus cost in the placebo group) was $£ 277$ ( $95 \% \mathrm{CI},-180$ to 783 ), or approximately $\$ 425$, and the difference in societal costs was $€ 34$ (95\% CI, -582 to 655 ), or approximately $\$ 50$.

Of the 281 recurrences during the trial, 58 re- 


\begin{tabular}{|c|c|c|c|c|}
\hline Variable & $\begin{array}{l}\text { Recurrence of Cellulitis } \\
\text { no. of events/total no. } \\
\text { of patients (\%)* }\end{array}$ & $\begin{array}{l}\text { Percentage-Point } \\
\text { Difference } \\
\text { (95\% Cl) }\end{array}$ & $\begin{array}{l}\text { Hazard Ratio } \\
(95 \% \mathrm{Cl})\end{array}$ & P Value \\
\hline \multicolumn{5}{|c|}{ Primary analysis: prophylaxis phase, year 1} \\
\hline Penicillin & $30 / 136(22)$ & $-15(-26$ to -4$)$ & $0.55(0.35$ to 0.86$)$ & 0.01 \\
\hline Placebo & $51 / 138(37)$ & & & \\
\hline \multicolumn{5}{|c|}{$\begin{array}{l}\text { Secondary analysis: follow-up phase, years } 2 \\
\text { and } 3 \uparrow\end{array}$} \\
\hline Penicillin & $26 / 97(27)$ & $0(-14$ to 12$)$ & $1.08(0.61$ to 1.93$)$ & 0.78 \\
\hline Placebo & $22 / 81(27)$ & & & \\
\hline
\end{tabular}

* The proportion of patients with a recurrence of cellulitis was a prespecified secondary end point. Proportions are presented as percentages, not person-time event rates.

$\uparrow$ The secondary analysis for years 2 and 3 was postrandomization. As a result, the groups may not have been balanced at the start of this period.

sulted in hospital admission (30 recurrences in the penicillin group and 28 in the placebo group). The mean length of the hospital stay was 10.0 days in the penicillin group and 9.2 days in the placebo group.

\section{DISCUSSION}

These data show that recurrent cellulitis is common among patients who have previously had two or more episodes. In the placebo group, 53\% of participants had at least one recurrence during the 3-year trial. Low-dose prophylactic penicillin given for a period of 12 months almost halved the risk of recurrence during the intervention period, and patients who received prophylaxis had significantly fewer recurrent episodes over the 3 -year period than those who received placebo. Although some level of protection appeared to be sustained for several months after the end of prophylactic therapy, this effect was lost by 36 months, a finding that suggests that longer-term prophylaxis may be required. Exactly how long such prophylaxis should be given is unclear and remains a matter of clinical judgment, depending on whether any predisposing factors, such as broken skin or lymphedema, can be adequately treated.

Results of adjusted analyses suggest that patients with a BMI of 33 or higher, multiple previous episodes of cellulitis, or lymphedema of the leg had a reduced likelihood of a response to prophylaxis. Because such patients are most likely to receive long-term prophylaxis, ${ }^{8}$ this finding warrants further investigation. The poor treatment

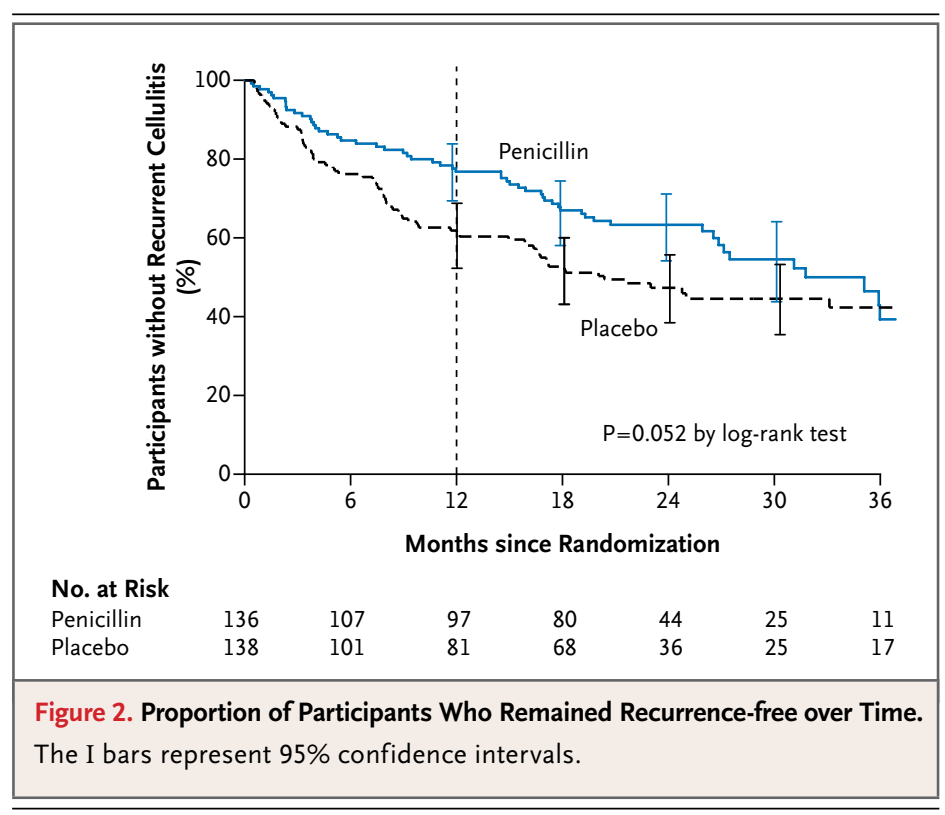

response in participants with a high BMI may mean that a higher dose of penicillin is required in these patients. Reducing edema in patients with leg lymphedema may also be important.

The methods used in this trial are similar to those used in our previous trial, which investigated a shorter duration of treatment (6 months of penicillin) in 123 patients with either a first episode of cellulitis (79\%) or recurrent cellulitis (21\%). Although in that trial, the reduction in the risk of recurrence with low-dose penicillin as compared with placebo was not significant (hazard ratio, $0.53 ; 95 \% \mathrm{CI}, 0.26$ to $1.07 ; \mathrm{P}=0.08$ ), the magnitude of the effect was similar. A Cochrane 


\begin{tabular}{|c|c|c|}
\hline Factor & $\begin{array}{l}\text { Odds Ratio } \\
(95 \% \mathrm{Cl}) \dagger\end{array}$ & P Value \\
\hline \multicolumn{3}{|l|}{$\begin{array}{l}\text { No. of previous cellulitis } \\
\text { episodes }\end{array}$} \\
\hline$\geq 3$ & $3.23(1.82-5.73)$ & $<0.001$ \\
\hline$<3$ & 1 & \\
\hline \multicolumn{3}{|l|}{ Edema } \\
\hline Preexisting edema & $1.83(0.97-3.47)$ & 0.06 \\
\hline No evidence of edema & 1 & \\
\hline \multicolumn{3}{|l|}{ BMI } \\
\hline$\geq 33$ & $2.05(1.16-3.64)$ & 0.01 \\
\hline$<33$ & 1 & \\
\hline
\end{tabular}

* Failure of prophylaxis was defined as at least one confirmed episode of cellulitis during the prophylaxis phase. $\dagger$ All effects were included in the model; data for two patients were not included owing to a missing value for BMI.

systematic review of the prevention of cellulitis is currently under way. ${ }^{20}$

This was a pragmatic trial that was designed to reflect normal clinical care. However, regular telephone contact between the coordinating center and participants may have increased rates of adherence to the study medications.

This trial highlights the need for long-term follow-up in studies of preventive treatment. Slower-than-expected recruitment meant that com- plete follow-up data were not available for all participants, and findings beyond 18 months should be interpreted cautiously.

The main cause of cellulitis is group A streptococci, for which resistance to penicillin is not an issue at this time. ${ }^{21}$ However, because microbiologic samples were not collected during the trial, the effect of prophylaxis on bacterial resistance more generally is not clear. Future studies that include collection of microbiologic samples would be useful.

In conclusion, this trial provides evidence that patients with two or more episodes of leg cellulitis who are given prophylactic penicillin for 12 months have fewer recurrences than those given placebo, without any increase in adverse effects. Patients with a high BMI, preexisting edema, or at least three episodes of previous cellulitis were less likely to have a response to prophylaxis than other patients. It is unclear how long prophylaxis should be continued.

Supported by a grant from Action Medical Research (SP4063).

Disclosure forms provided by the authors are available with the full text of this article at NEJM.org.

We thank the late Professor Neil Cox, who conceived and designed the trial in collaboration with members of the U.K. Dermatology Clinical Trials Network; the National Institute for Health Research, which (through the Comprehensive Local Research Network) provided research nurse support; the Nottingham Clinical Trials Unit, which provided the randomization service and support with data management; and the Clinical Trials Pharmacists at Nottingham University Hospitals National Health Service Trust, who provided pharmacy arrangements.
REFERENCES

1. Cox NH. Oedema as a risk factor for multiple episodes of cellulitis/erysipelas of the lower leg: a series with community follow-up. Br J Dermatol 2006;155:947-50. 2. Thomas K, Crook A, Foster K, et al. Prophylactic antibiotics for the prevention of cellulitis (erysipelas) of the leg: results of the U.K. Dermatology Clinical Trials Network's PATCH II trial. Br J Dermatol 2012;166:169-78.

3. Jeng A, Beheshti M, Li J, Nathan R. The role of beta-hemolytic streptococci in causing diffuse, nonculturable cellulitis: a prospective investigation. Medicine (Baltimore) 2010;89:217-26.

4. Bernard P, Bedane C, Mounier M, Denis F, Catanzano G, Bonnetblanc JM. Streptococcal cause of erysipelas and cellulitis in adults: a microbiologic study using a direct immunofluorescence technique. Arch Dermatol 1989;125:779-82.

5. Damstra RJ, van Steensel MAM, Boomsma JHB, Nelemans P, Veraart JCJM. Erysipelas as a sign of subclinical primary lymphoedema: a prospective quantitative scintigraphic study of 40 patients with unilateral erysipelas of the leg. Br J Dermatol 2008;158:1210-5.

6. Soo JK, Bicanic TA, Heenan S, Mortimer PS. Lymphatic abnormalities demonstrated by lymphoscintigraphy after lower limb cellulitis. Br J Dermatol 2008;158:1350-3. 7. Levell NJ, Wingfield CG, Garioch JJ. Severe lower limb cellulitis is best diagnosed by dermatologists and managed with shared care between primary and secondary care. Br J Dermatol 2011;164:1326-8.

8. Guidelines on the management of cellulitis in adults. Belfast, Northern Ireland: Clinical Resource Efficiency Support Team, 2005 (http://www.acutemed.co.uk/ docs/Cellulitis\%20guidelines,\%20CREST, \%2005.pdf).

9. Consensus document on the management of cellulitis in lymphoedema. Oxfordshire, United Kingdom: British Lymphology Society, 2010 (http://www.thebls .com/docs/consensus.pdf).

10. Kremer M, Zuckerman R, Avraham Z, Raz R. Long-term antimicrobial therapy in the prevention of recurrent sort-tissue infections. J Infect 1991;22:37-40.
11. Sjöblom AC, Eriksson B, JorupRönström C, Karkkonen K, Lindqvist M. Antibiotic prophylaxis in recurrent erysipelas. Infection 1993;21:390-3.

12. Chakroun M, Ben-Romdhane F, Battikh R, Souki A, Bouzouaia N. Benzathine penicillin prophylaxis in recurrent erysipelas. Med Mal Infect 1994;24:894-7. (In French.) 13. Cox NH, Colver GB, Paterson WD. Management and morbidity of cellulitis of the leg. J R Soc Med 1998;91:634-7.

14. Jorup-Rönström C, Britton S. Recurrent erysipelas: predisposing factors and costs of prophylaxis. Infection 1987;15:105-6.

15. Grambsch PM, Therneau TM. Proportional hazards tests and diagnostics based on weighted residuals. Biometrika 1994;81:515-26.

16. Prescription cost analysis - England, 2010. Leeds, United Kingdom: National Health and Social Care Information Centre, National Health Service, 2011 (http://www .ic.nhs.uk/pubs/prescostanalysis2010).

17. Unit costs of health and social care 2010. Kent, United Kingdom: Personal Social Services Research Unit University 
of Kent, 2010 (www.pssru.ac.uk/pdf/uc/ uc2010/uc2010.pdf).

18. National schedule of reference costs 2010-11 for NHS Trusts and PCTs. London: Department of Health, National Health Service, 2011 (http://www.dh.gov.uk/en/ Publicationsandstatistics/Publications/ PublicationsPolicyAndGuidance/ DH_131140).
19. Barber JA, Thompson SG. Analysis of cost data in randomized trials: an application of the non-parametric bootstrap. Stat Med 2000;19:3219-36.

20. Dalal A, Eskin-Shwartz M, Mimouni $\mathrm{D}$, et al. Interventions for the prevention of recurrent erysipelas and cellulitis. Cochrane Database Syst Rev 2012;4: CD009758.
21. Resistance surveillance website: bacteraemia search results. Birmingham, United Kingdom: British Society for Antimicrobial Chemotherapy, 2011 (http://www.bsacsurv .org/mrsweb/bacteraemia?organism=A\& antimicrobial=All\&year $=$ All\&country $=$ All \&summary=SIRSummary\&formname= bsac_bacteraemia\&submit=Search). Copyright (@) 2013 Massachusetts Medical Society.

The New England Journal of Medicine 